\title{
Ferromagnetic Spin Systems at Low Temperatures
}

\author{
J. Slawny \\ Joseph Henry Laboratories of Physics, Princeton University, Princeton, New Jersey 08540, USA
}

\begin{abstract}
Finite-spin systems with ferromagnetic, finite range interactions are considered. Using Ruelle's theorem on zeros of polynomials contracted according to Asano, analyticity of pressure and correlation functions is proved. A description of all translation invariant equilibrium states at low temperatures for a large class of systems is given.
\end{abstract}

\section{Introduction}

We develop here further the technique of $[13,14]$. In combination with the results of [6] it allows us to complete a picture of classical spin systems with ferromagnetic interactions at low temperatures.

If $J$ is any finite range ferromagnetic interaction one associates with it a family $\mathfrak{U}(J)$ of functions on the configuration space and proves that for low enough temperatures all translation invariant equilibrium states agree on elements of this family.

Furthermore, the symmetriy group $\mathscr{S}$ is introduced. It acts on the configuration space of the system by flipping spins at lattice sites in such a way that leaves the energy invariant. Let, for $G \in \mathscr{S}, \varrho_{G}^{+}$be the (equilibrium) state $f \mapsto \mapsto \varrho^{+}(f \circ G),{ }^{1}$ and for any probability measure $\mu$ on $\mathscr{S}$ let

$$
\varrho_{\mu}=\int_{\mathscr{S}} \varrho_{G}^{+} \mu(d G) \text {. }
$$

The group $\mathscr{S}(J)$ and the family $\mathfrak{A}(J)$ are closely related: the closed linear span of $\mathfrak{A}(J)$ consists exactly of all $\mathscr{S}$-invariant functions. From the uniqueness on $\mathfrak{U}(J)$ we weduce - this is our main result - that for any ferromagnetic spin system with finite range interaction all $\mathbb{Z}^{\nu}$-invariant equilibrium states at low enough temperature have the integral representation $(0.1)$.

The representation is made unique by intergrating over $\mathscr{S} / \mathscr{S}^{+}$instead of $\mathscr{S}$ where $\mathscr{S}^{+}$is the isotropy subgroup of $\varrho^{+} \cdot \mathbb{Z}^{v}$ acts on $\mathscr{S} / \mathscr{S}^{+}$in a natural way, and $\varrho_{\mu}$ is $\mathbb{Z}^{\nu}$-invariant iff $\mu$ is, $\varrho_{\mu}$ is ergodic iff $\mu$ is. Thus the description of all invariant equilibrium states at low temperatures is reduced, in a sense, to finding $\mathscr{S} / \mathscr{S}^{+}$

* $\quad$ Research supported in part by AFOSR F 44620 71-C-0108 and by US NSF Grant GP 39048.

$1 \varrho^{+}$is the equilibrium state defined by fixing the maximal spin outside $\Lambda$ and letting $\Lambda \nearrow \infty$, Section 2.1 
and to describing $\mathbb{Z}^{v}$-ergodic measures on this group; we consider this problem in Section 5.

Most of the results of this paper are obtained by first reducing to the spin $\frac{1}{2}$ case by a method due to Griffiths. Then the analyticity of pressure, needed in the proof of uniqueness on $\mathfrak{A}(J)$, is demonstrated using Ruelle's theorem on zeros of polynomials contracted according to Asano; and in an unpublished proof of the results of [6], which are used in Section 5 in a discussion of $\mathscr{S} / \mathscr{S}^{+}$, an extension of Peierls' argument is employed.

In applying the Asano contraction method to the problems of the present paper we make extensive use of the connection between the Asano decomposition and a decomposition property of a group appearing in High Temperature Expansion; this connection was noticed earlier [13]. The decomposition property of the group - Theorem 3.5 below - is the single most difficult result needed here. In full generality, as used in this paper, we owe this decomposition theorem to Holsztyński [5]; we proved it for a class of systems in [13].

In more detail, the content of the present paper is as follows. Section 1: definitions and properties of equilibrium states, Griffiths' representation of systems of arbitrary spin in terms of spin $\frac{1}{2}$ systems and some inequalities. Section 2: definition and properties of the state $\varrho^{+}$, the symmetry group $\mathscr{S}$, the subgroup $\mathscr{S}^{+}$, the integral representation. Section 3: Low Temperature Expansion, the groups $\Gamma$ and $\mathscr{K}$, Ruelle's theorem on Asano contractions, Asano contractions and a decomposition property of $\mathscr{K}$, Holsztyński's theorem. Section 4: analyticity properties of pressure, the analyticity and uniqueness theorem, zero-temperature limit of the entropy and states. Section 5: determination of ergodic equilibrium states in a number of cases, theorem on $\mathscr{S} / \mathscr{S}^{+}$for systems in $\mathbb{Z}^{v}$, criterion of the finiteness of the number of ergodic equilibrium states at low temperatures, factorizable systems. Section 6: remarks.

A number of papers on application of Asano contractions technique through Ruelle's theorem is listed in $[13]^{2}$; cf. also [20,21, 22]. For references to papers on Low and High Temperature Expansions we refer to [13,14]. An Ising model version of [13, Lemma 6] appeared in [23].

As noted in [13 Sec. 4.10$]$ most of the results admit a generalization to systems with interactions dominated by their ferromagnetic part; we omit here the precise formulation.

\section{Equilibrium States}

1.1. Let $m$ be a positive integer and let $\mathbb{L}$ be a denumerable set. A configuration of spin $\frac{m}{2}$ classical system on $\mathbb{L}$ is a function from $\mathbb{L}$ to $\{-m,-m+2, \ldots, m-2, m\}$. The set

$$
\mathscr{X}=\{-m,-m+2, \ldots, m\}^{\mathbb{L}},
$$

we write sometimes $\mathscr{X}_{\mathbb{L}}$, of all configurations is made into a compact separable space by the product topology. $s_{a}, a \in \mathbb{L}$, will denote the function on $\mathscr{X}$ which to each configuration assigns its value at the point $a$.

\footnotetext{
2 See especially $[15,10]$. For finite systems the contour homomorphism $\gamma$ and the group $\overline{\mathscr{B}}$ were introduced in $[10]$.
} 
A multiplicity function (m.f.), [4], is a function from $\mathbb{L}$ to $\{0,1,2, \ldots\}$, zero at almost all points. For a m.f. $A$ we define

$$
\begin{aligned}
\operatorname{supp}(A) & =\{a \in \mathbb{L}: A(a) \neq 0\} \\
|A| & =\sum_{a \in \operatorname{supp}(A)} A(a)
\end{aligned}
$$

and

$$
s_{A}=\prod_{a \in \operatorname{supp}(A)} s_{a}^{A(a)} .
$$

When $m=1$ we write $\sigma_{a}$ instead of $s_{a}$. A m.f. $A$ is called even if $A(a)$ is an even number for all $a \in \operatorname{supp}(A)$. The addition of m.f.'s is defined pointwise:

$$
(A+B)(a)=A(a)+B(a),
$$

so that

$$
S_{A} \cdot S_{B}=S_{A+B} .
$$

An interaction, say $J$, is a real valued function on the set of m.f.'s such that for any $a \in \mathbb{L}$

$$
\sum_{B: a \in \operatorname{supp}(B)} m^{|B|}|J(B)|<\infty .
$$

The support of $J$ is denoted by $\mathscr{B}$, or $\mathscr{B}(J)$, and its elements are called bonds. Interactions form a vector space over $\mathbb{R}$.

Let $\Lambda$ be a finite subset of $\mathbb{L}$. The Gibbs state in $\Lambda$ corresponding to a configuration $Y$ in $\mathbb{L} \backslash A$, to an interaction $J$ and to a temperature $\beta^{-1}$ ascribes to a configuration $X$ on $\Lambda$ the probability

$$
\varrho_{\Lambda}^{Y}(X)=\left(Z_{\Lambda}^{Y}\right)^{-1} \exp \sum_{\operatorname{supp}(B) \cap \Lambda \neq \emptyset} K(B) S_{B}(X, Y)
$$

where $K(B)=\beta J(B)$,

$$
Z_{A}^{Y}=\sum_{X \in \mathscr{X}_{A}} \exp \sum_{\operatorname{supp}(B) \cap A \neq \emptyset} K(B) s_{B}(X, Y)
$$

and $X, Y$ is the configuration in $\mathbb{L}$ equal to $X$ in $\Lambda$ and to $Y$ in $\mathbb{L} \backslash \Lambda$.

Let $\mathfrak{A}$ be the $C^{*}$-algebra of complex continuous functions on $\mathscr{X}$ and let $\mathfrak{U}_{\Lambda}$ be the subalgebra of the functions depending on the restrictions of configurations to $\Lambda$ only; $\mathfrak{I}_{\Lambda}$ will be identified with $\mathscr{C}\left(\mathscr{X}_{\Lambda}\right)$.

A probability measure on $\mathscr{X}$, or, equivalently, a state of $\mathfrak{U}$ is called an equilibrium state (corresponding to $K$ ) if when restricted to $\mathfrak{U}_{\Lambda}, \Lambda$ finite, it is a combination of the states $\left\{\varrho_{A}^{Y}\right\}_{Y}$ :

$$
\varrho(f)=\int \varrho_{\Lambda}^{Y}(f) \varrho_{\Lambda}(d Y), \quad f \in \mathfrak{H}_{\Lambda},
$$

where $\tilde{\varrho}_{\Lambda}$ is a measure on $\mathscr{X}_{\mathbb{L} \backslash \Lambda}$.

The set of all equilibrium states is dentoed by $\Delta$; we write $\Delta_{K}$ or $\Delta_{J, \beta}$ when discussing the dependence of $\Delta$ on the interaction and $\beta . \Delta$ is a compact separable space when equipped with the $\omega^{*}$-topology, which in our case is defined by the family of functions $\varrho \mapsto \varrho\left(s_{A}\right), A$ running through the set of all m.f.'s. ${ }^{3} \Delta$ is a Choquet

3 In fact any $s_{A}$ is a linear combination of $s_{A}$, with $\operatorname{supp}\left(A^{\prime}\right) \subset \operatorname{supp}(A)$ and $A^{\prime}(a) \leqq m[4$, footnote 8] but we will not use this reduction. 
simplex, i.e. for each $\varrho \in \Delta$ there exists (unique) measure carried by the set $\mathscr{E}(\Delta)$ of the extremal points of $\Delta$ with the resultant $\varrho{ }^{4}$

1.2. $\mathbb{L}$ will be called a ( $v$-dimensional) lattice if it is a discrete $\mathbb{Z}^{v}$ invariant subset of $\mathbb{R}^{v}$. Since the injection of $\mathbb{L}$ in $\mathbb{R}^{v}$ will be of no importance in what follows we could define a lattice as a free $\mathbb{Z}^{v}$-space with a finite number of orbits. We write $a+x$ or $\tau_{x}(a)$ for the translate of $a \in \mathbb{L}$ by $x \in \mathbb{Z}^{v}$.

The set of translation invariant equilibrium states is denoted by $\Delta^{I} ; \Delta^{I}$ is also a Choquet simplex. Considering the induced action of $\mathbb{Z}^{v}$ on the set of m.f.'s we call a subfamily $\mathscr{B}_{0}$ of $\mathscr{B}$ fundamental if each bond is congruent with exactly one element of $\mathscr{B}_{0}$. Similarly one defines a fundamental subset for any $\mathbb{Z}^{v}$-space, as for instance $\mathbb{L}$.

Let the interaction be translation invariant and let

$$
Z_{A}=\sum_{x \in \mathscr{X}_{A}} \exp \sum_{\operatorname{supp}(B) \subset A} K(B) s_{B}(X) .
$$

The pressure

$$
p(K)=\lim _{\Lambda \rightarrow \infty} \frac{1}{|\Lambda|} \log Z_{\Lambda}
$$

exists and is a convex function of $K$; here the limit is understood in the Van Hove sense. $^{5}$

For any translation invariant state $\varrho$ the mean energy

$$
\lim _{\Lambda \rightarrow \infty} \frac{1}{|\Lambda|} \varrho\left(-\sum_{\operatorname{supp}(B) \subset A} K(B) S_{B}\right)
$$

is equal to $-\varrho\left(\sum_{B \in \mathscr{B}_{0}} K(B) S_{B}\right)$ where $\mathscr{B}_{0}$ is any fundamental family of bonds. A translation invariant state on $\mathfrak{U}$ is a translation invariant equilibrium state if and only if

$$
p(K)=s(\varrho)+\varrho\left(\sum_{B \in \mathscr{B}_{0}} K(B) s_{B}\right)
$$

where $s(\varrho)$ is the entropy of the state $\varrho[8]$.

1.3. Proposition. Let $A$ be a $m$.f. and let the interaction $K_{A}$ be defined by

$$
K_{A}(B)= \begin{cases}1 & \text { if } B \text { is a translate of } A \\ 0 & \text { otherwise } .\end{cases}
$$

If the function $\lambda \rightarrow p\left(K+\lambda K_{A}\right)$ is differentiable at zero then $\varrho\left(s_{A}\right)$ has the same value for all $\varrho \in \Delta_{K}^{I}$ and

$$
\varrho\left(s_{A}\right)=\left.\frac{d}{d \lambda} p\left(K+\lambda K_{A}\right)\right|_{\lambda=0} .
$$

One can prove this proposition as indicated in [13, Section 1.4]. Another proof, which yields corresponding result also for quantum lattice systems can be based on [19]. Still another, a more direct one, will presumably appear in [7].

4 We refer to [1], [8] for the notion and properties of equilibrium states.

5 The usual definition of the Van Hove limit ([11], Section 2.1), and the theorem on the convergence of pressure and entropy generalizes easily to our case. In fact we will use very regular nets only (Theorem 4.5). 
1.4. Our results for arbitrary spin are obtained through Griffiths' representation by spin $\frac{1}{2}$ systems:

For any set IL define

$$
\mathbb{L}^{m}=\mathbb{L} \times\{1, \ldots, m\}, \mathscr{X}^{m}=\{1,-1\}^{\mathbb{I}^{m}},
$$

and for any m.f. $B$ on IL

$$
\sigma_{B^{m}}=\prod_{a \in \mathbb{L}}\left(\sigma_{a, 1}+\ldots+\sigma_{a, m}\right)^{B(a)} ;
$$

$\sigma_{B^{m}}$ is a function on $\mathscr{X}^{m}$.

Let $\Lambda$ be a finite set and let $K$ be an interaction of a spin $\frac{m}{2}$ system in $\Lambda$. It was shown in [4] that the partition function

$$
Z=\sum_{X_{\in} \mathscr{X}_{A}} \exp \sum_{B} K(B) S_{B}(X)
$$

is equal to

$$
\sum_{X \in \mathscr{X}_{\Lambda}^{m}} \exp \left[\sum_{B} K(B) \sigma_{B^{m}}+\sum_{a \in \Lambda} \sum_{r, s=1}^{m} K_{r, s}\left(\sigma_{a, r} \sigma_{a, s}-1\right)\right](X)
$$

with suitably chosen $K_{r, s}$; a possible choice is

$$
\begin{array}{cl}
K_{r, s}=\frac{1}{2} \log (r+1) & \text { if } s=r+1, r=1, \ldots, m-1 \\
0 & \text { otherwise } .
\end{array}
$$

Similarly, for the (magnetic) correlation functions

$$
\varrho\left(s_{A}\right)=Z^{-1} \sum_{X \in \mathscr{X}_{A}} s_{A}(X) \exp \sum_{B} K(B) s_{B}(X)
$$

one has the formula

$$
\begin{aligned}
\varrho\left(s_{A}\right)= & Z^{-1} \sum_{X \in \mathscr{X}_{A}^{m}} \sigma_{A} m(X) \exp \left[\sum_{B} K(B) \sigma_{B^{m}}\right. \\
& \left.+\sum_{a \in A} \sum_{r, s-1}^{m} K_{r, s}\left(\sigma_{a, r} \sigma_{a s}-1\right)\right](X) .
\end{aligned}
$$

If the original interaction is ferromagnetic, the resulting spin $\frac{1}{2}$ system is ferromagnetic too.

From (1.6) and corresponding inequalities for spin $\frac{1}{2}$ systems, it follows, [4, 3], that if $K(B) \geqq 0$ then for all m.f.'s $A, B$

$$
\begin{gathered}
\varrho\left(s_{A}\right) \geqq 0 \\
\varrho\left(s_{A} s_{B}\right) \geqq \varrho\left(s_{A}\right) \varrho\left(s_{B}\right) .
\end{gathered}
$$

If $K^{\prime}$ is any interaction such that $\left|K^{\prime}(A)\right| \leqq K(A)$ for any m.f. $A$ then the Gibbs state $\varrho^{\prime}$ corresponding to $K^{\prime}$ is majorized by the one corresponding to $K$ in the sense that

$$
\varrho^{\prime}\left(s_{A}\right) \leqq \varrho\left(s_{A}\right) \quad \text { for any m.f. } \quad A .
$$

This follows from the corresponding inequality for spin $\frac{1}{2}$ systems [14, Formula (G.2)] via (1.6).

6 Here and in what follows it is assumed that $\sum_{\text {supp }(B) \in a} m^{|B|}|K(B)|<\infty$. 
We will need later also the following generalization of the inequality (4.19) of [4]: For a ferromagnetic interaction $K$ define two interactions of spin $\frac{1}{2}$ system on $\Lambda$ by

$$
\bar{K}(C)=\sum_{\bar{B}=C} m^{|B|} K(B) \quad \text { and } \quad \tilde{K}(C)=\sum_{\bar{B}=C} \tilde{m}^{|B|} K(B),
$$

where $\tilde{m}$ is equal to $\frac{m}{2}$ for even $m$, and $\frac{m+1}{2}$ for odd $m$. Then

$$
\tilde{m}^{|A|} \varrho \tilde{K}\left(\sigma_{\bar{A}}\right) \leqq \varrho\left(s_{A}\right) \leqq m^{|A|} \varrho_{\bar{K}}\left(\sigma_{\bar{A}}\right) ;
$$

here $\bar{A}$ is the characteristic function of $\{a \in \Lambda: A(a)$ is odd $\}$ and $\varrho_{\bar{K}}, \varrho_{\widetilde{K}}$ are the Gibbs states of $\operatorname{spin} \frac{1}{2}$ systems on $\Lambda$ with interactions $\bar{K}, \tilde{K}$.

Inequality (1.9) can be slightly sharpened but it is strong enough to yield our results on the low temperature behaviour.

\section{The State $\varrho^{+}$and the Integral Representation}

In this section we consider spin $\frac{m}{2}$ systems on a denumerable set $\mathbb{L}$ with a ferromagnetic interaction satisfying the condition (1.1).

2.1. It is easy to see that the state $\varrho_{\Lambda}^{Y}$ fits into the framework of Section 1.4 with an interaction $K^{Y}$ in $\Lambda$ depending on the configuration $Y$ outside of $\Lambda$. If $\varrho_{\Lambda}^{+}$corresponds to $Y_{a}=m, a \in \mathbb{L} \backslash \Lambda$, and $K^{+}$is the corresponding interaction then for any configuration $Y$ outside of $\Lambda$ and any m.f. $B$ in $\Lambda$

$$
\left|K^{Y}(B)\right| \leqq K^{+}(B) .
$$

Therefore, by (1.8),

$$
\varrho_{\Lambda}^{+}\left(s_{A}\right) \geqq \varrho_{\Lambda}^{Y}\left(s_{A}\right), \forall Y, A,
$$

and by the definition of equilibrium state

$$
\varrho_{A}^{+}\left(s_{A}\right) \geqq \varrho\left(s_{A}\right), \quad \forall \varrho \in \Delta
$$

for any m.f. $A$ in $\Lambda$.

From (2.1), (2.2), and (1.7) one deduces (like, for instance, in [14]) that $\varrho_{A}^{+}\left(s_{A}\right)$ converges as $\Lambda \rightarrow \infty$ and that the limits define an equilibrium state, denoted $\varrho^{+}$, with the following properties:

$$
\varrho^{+}\left(s_{A}\right) \geqq \varrho\left(s_{A}\right), \quad \forall \varrho \in \Delta, \forall A
$$

and

$$
\varrho^{+}\left(s_{A} s_{B}\right) \geqq \varrho^{+}\left(s_{A}\right) \varrho^{+}\left(s_{B}\right), \quad \forall A, B .
$$

It follows from (2.3) (cf. [14]) that $\varrho^{+}$is an extremal equilibrium state and that it is invariant under any transformation of $\mathbb{L}$ leaving the interaction invariant.

2.2. The Symmetry Group. We define an action of the compact, separable, abelian group

$$
\mathscr{G}=\{1,-1\}^{\mathbb{L}}
$$


on the space $\mathscr{X}$ of configurations: if $G=\left(G_{a}\right)_{a \in \mathbb{L}} \in \mathscr{G}$ and $X=\left(X_{a}\right)_{a \in \mathbb{L}} \in \mathscr{X}$ then

$$
(G X)_{a}=G_{a} X_{a}
$$

$G$ is flipping the $a$-th coordinate of the configuration if $G_{a}=-1$. Obviously, the mapping $\mathscr{G} \times \mathscr{X} \ni(G, X) \rightarrow G X \in \mathscr{X}$ is continuous.

The dual group to $\mathscr{G}$ will be identified with $\mathscr{P}_{f}(\mathbb{L})$ (equipped with the discrete topology $)^{7}$ : the character $\sigma_{A}$ of $\mathscr{G}$ corresponding to $A \in \mathscr{P}_{f}(\mathbb{L})$ is defined by

$$
\sigma_{A}(G)=\prod_{a \in A} G_{a} .
$$

If $A$ is a.m.f. then we define an element $\bar{A}$ of $\mathscr{P}_{f}(\mathbb{L})$.

$$
\bar{A}=\{a \in L: A(a) \text { is odd }\},
$$

and if $\mathscr{B}$ is a family of m.f.'s then $\overline{\mathscr{B}}$ will denote the subgroup of $\mathscr{P}_{f}(\mathbb{L})$ generated by $\{\bar{B}: B \in \mathscr{B}\}$; this notation agrees with that employed in [14] in the spin $\frac{1}{2}$ case. We note that for any m.f. $A$ and any $G \in \mathscr{G}$

$$
s_{A}^{\circ} G=\sigma_{\bar{A}}(G) s_{A} .
$$

For any state $\varrho$ of $\mathfrak{U}$ (i.e. any probability measure on $\mathscr{X}$ ) we denote by $\varrho_{G}$ the state

$$
f \mapsto \varrho(f \circ G) ;
$$

by $(2.7)$

$$
\varrho_{G}\left(s_{A}\right)=\sigma_{\bar{A}}(G) \varrho\left(s_{A}\right) .
$$

The mapping $G \mapsto \varrho_{G}$ is continuous when the set of states is equipped with the $\omega^{*}$-topology.

The symmetry group $\mathscr{S}$ of the system:

$$
\mathscr{S}=\left\{G \in \mathscr{G}: s_{B} \circ G=s_{B}, \forall B \in \mathscr{B}\right\}
$$

is a closed subgroup of $\mathscr{G}$ and therefore compact. By (2.7),

$$
\mathscr{S}=\left\{G \in \mathscr{G}: \sigma_{B}(G)=1, \forall B \in \overline{\mathscr{B}}\right\} .
$$

For $G \in \mathscr{G}, \Lambda \subset \mathbb{L}$ and for $Y \in \mathscr{X}_{\Lambda}$ let $(G Y)_{a}=G_{a} Y_{a}, a \in \Lambda$. If

$$
\varrho(f)=\int_{\mathscr{X} \mathbb{L} \backslash \Lambda} \varrho_{\Lambda}^{Y}(f) \varrho_{\Lambda}(d Y), \quad f \in \mathfrak{U}_{\Lambda},
$$

then, as is not hard to see,

$$
\varrho_{G}(f)=\int_{\mathscr{X} \backslash \Lambda} \varrho_{\Lambda}^{G Y} \tilde{\varrho}_{\Lambda}(d Y)
$$

for any $G \in \mathscr{S}$. Hence $\mathscr{S}$ is leaving invariant the set $\Delta_{K}$ of equilibrium states. Since the mapping $\varrho \mapsto \varrho_{G}$ preserves convex combinations $\varrho_{G}$ is an extremal equilibrium state if $\varrho$ is.

$7 \quad$ For any set $\mathbb{L}, \mathscr{P}(\mathbb{L})$ denotes the (abelian) group of all subsets of $\mathbb{L}$ with symmetric difference of sets as the group operation. $\mathscr{P}_{f}(\mathbb{L})$ is the subgroup of all finite subsets of $\mathbb{L}$. 
For a probability measure $\mu$ on $\mathscr{S}$ we define

$$
\varrho_{\mu}=\int \varrho_{G}^{+} \mu(d G)
$$

(2.8) yields

$$
\varrho_{\mu}\left(s_{A}\right)=\mu\left(\sigma_{\bar{A}}\right) \varrho^{+}\left(s_{A}\right) .
$$

Let $\mathscr{S}^{+}$be the isotropy subgroup of $\varrho^{+}$:

$$
\mathscr{S}^{+}=\left\{G \in \mathscr{S}: \varrho_{G}^{+}=\varrho^{+}\right\} \text {. }
$$

The function $G \mapsto \varrho_{G}^{+}$is constant on $\mathscr{S}^{+}$-cosets. Therefore the integral in (2.11) can be transformed into an integral over $\mathscr{S} / \mathscr{S}^{+}$or, equivalently, into an integral over $\mathscr{S}$ with respect to a $\mathscr{S}^{+}$-invariant measure. $[G] \rightarrow \varrho_{[G]}^{+}$will denote the function on $\mathscr{S} / \mathscr{S}^{+}$corresponding to $G \mapsto \varrho_{G}^{+}$; by the definition of $\mathscr{S}^{+},[G] \rightarrow \varrho_{[G]}^{+}$is an injective mapping. We write

$$
\varrho_{\mu}=\int \varrho_{[G]}^{+} \mu(d[G])
$$

for the integral over $\mathscr{S} / \mathscr{S}^{+}$that corresponds to (2.11).

Let

$$
\mathscr{B}^{+}=\left\{A: \varrho^{+}\left(s_{A}\right) \neq 0\right\}
$$

By (2.4), $\mathscr{B}^{+}$is an additive family of m.f.'s. Therefore, the image of $\mathscr{B}^{+}$in $\mathscr{P}_{f}(\mathbb{L})$ is the group $\overline{\mathscr{B}^{+}}$. It is not hard to deduce from (1.9) (cf. [14], inequality (6c)) that $\mathscr{B}^{+} \supset \mathscr{B}$. Hence $\overline{\mathscr{B}^{+}} \supset \overline{\mathscr{B}}$. We also note that by $(2.7)$ and $(2.8)$

$$
\begin{aligned}
& \mathscr{S}^{+}=\left\{G \in \mathscr{S}: s_{A} \circ G=s_{A}, \forall A \in \mathscr{B}^{+}\right\} \\
& \mathscr{S}^{+}=\left\{G \in \mathscr{S}: \sigma_{A}(G)=1, \forall A \in \overline{\mathscr{B}^{+}}\right\}
\end{aligned}
$$

If the interaction is invariant under a group of tranformations of $\mathbb{L}$ then $\mathscr{S}, \varrho^{+}$ and therefore also $\mathscr{S}^{+}$are invariant under this group. Thus we can consider the action of the group on $\mathscr{S} / \mathscr{S}^{+}$.

Let

$$
\Delta^{+}=\left\{\varrho \in \Delta: \varrho\left(s_{A}\right)=\varrho^{+}\left(s_{A}\right) \text { if } \quad \bar{A} \in \overline{\mathscr{B}}\right\} .
$$

Obviously, $\Delta^{+}$is a convex and closed subset of $\Delta$, and all states $\varrho_{\mu}$ are in $\Delta^{+}$.

2.3. Theorem. All elements of $\Delta^{+}$are of the form (2.14). The mapping $\mu \rightarrow \varrho_{\mu}$ from probability measures on $\mathscr{S} / \mathscr{S}^{+}$to equilibrium states is one-to-one, and (2.14) gives the decomposition of $\varrho_{\mu}$ into extremal elements of $\Delta$. In case the interaction is invariant under a group of transformations of $\mathbb{L}, \mu$ is invariant if and only if $\varrho_{\mu}$ is invariant and $\mu$ is ergodic if and only if $\varrho_{\mu}$ is.

For a proof we refer to [14].

2.4. Sometimes it is more convenient to deal with the groups $\overline{\mathscr{B}^{+}}$and $\overline{\mathscr{B}}$ than with $\mathscr{S}^{+}$and $\mathscr{S}$. Then the following identification is useful.

$$
\mathscr{S} / \mathscr{S}^{+} \cong\left(\overline{\mathscr{B}^{+}} / \overline{\mathscr{B}}\right)^{\wedge} \text {. }
$$

8 The integral here is taken in the weak sense, i.e. $\varrho_{\mu}$ is the state $f \mapsto \int \varrho_{G}(f) \mu(d G)$ which is well defined by the continuity of $G \mapsto \varrho_{G}(f)$. 
This can be shown as follows: Formulas (2.10) and (2.15) can be also written as

$$
\mathscr{S}=\overline{\mathscr{B}}^{\lrcorner} \quad \text { and } \quad \mathscr{S}^{+}=\left(\overline{\mathscr{B}^{+}}\right)^{\perp}
$$

where the orthogonality is defined by the pairing

$$
G, A \rightarrow \sigma_{A}(G)
$$

Consider the induced pairing of $\mathscr{S}$ and $\overline{\mathscr{B}}{ }^{+}$. Corresponding homomorphisms $\mathscr{S} \rightarrow\left(\mathscr{\mathscr { B }}^{+}\right)^{\wedge}$ and $\mathscr{\mathscr { B }}^{+} \rightarrow \mathscr{S}^{\wedge}$ have $\mathscr{S}^{+}$and $\mathscr{\mathscr { B }}$ as kernels. Therefore one can define a pairing of $\mathscr{S} / \mathscr{S}^{+}$and $\overline{\mathscr{B}} \mathscr{\mathscr { B }}^{+} / \overline{\mathscr{B}}$ which separates points of both of these groups. Since the group $\mathscr{S} / \mathscr{S}^{+}$is compact and $\overline{\mathscr{B}}^{+} / \overline{\mathscr{B}}$ is discrete this yields, by well known theorem on duality for compact groups, the result (2.17).

In case the interaction is invariant under a group of transformations of $\mathbb{L}, \mathscr{S} / \mathscr{S}^{+}$is isomorphic to $(\overline{\mathscr{B}} / \overline{\mathscr{B}})^{\wedge}$ also as a group-space. When $\mathscr{S} / \mathscr{S}^{+}$is finite it can be identified with $\overline{\mathscr{B}}^{+} / \overline{\mathscr{B}}$, but in general not as group-spaces.

\section{3. $\operatorname{Spin} \frac{1}{2}$ and Asano Contractions}

The omitted proofs of the results described in this section are trivial modifications of corresponding parts of [13] or of papers of the reference list of [13].

3.1. In the spin $\frac{1}{2}$ case $\mathscr{X}=\mathscr{G}$. We consider only m.f.'s with values 0,1 and identify them with corresponding subsets of $\mathbb{L}$. We write then $\sigma_{A}$ instead of $s_{A}$, as in Section 2.2. In what follows it will be convenient to work with the slightly generalized framework of Sections 1.1-1.4 which we now formulate.

Let $\mathscr{B}$ be a set, whose elements will be called bonds, and let there be given a mapping $B \mapsto \sigma_{B}$ from $\mathscr{B}$ to characters of $\mathscr{X}$. Since the mapping $A \mapsto \sigma_{A}$ from $\mathscr{P}_{f}(\mathbb{L})$ to $\hat{\mathscr{X}}$ is bijective, for each $B \in \mathscr{B}$ there exists a unique finite subset $\bar{B}$ of $\mathbb{L}$ such that $\sigma_{B}=\sigma_{\bar{B}}$.

One defines homomorphisms

$$
\gamma: \mathscr{X} \rightarrow \mathscr{P}(\mathscr{B}), \gamma(X)=\left\{B \in \mathscr{B}: \sigma_{B}(X)=-1\right\},
$$

and $\beta \rightarrow \bar{\beta}$ from $\mathscr{P}_{f}(\mathscr{B})$ to $\mathscr{P}_{f}(\mathbb{L})$ extending the mapping $B \mapsto \bar{B}$. Let

$$
\Gamma=\operatorname{Im}(\gamma), \mathscr{K}=\operatorname{Ker}(\beta \mapsto \bar{\beta}), \mathscr{S}=\operatorname{Ker}(\gamma)
$$

An interaction is a function from $\mathscr{B}$ to real numbers. If $K$ is an interaction and $\lambda$ is a real number then the interaction $\lambda K$ is defined by $(\lambda K)(B)=\lambda K(B)$. If $K^{\prime}$ is another interaction then $K+K^{\prime}$ is the interaction with the disjoint sum of $\mathscr{B}$ and $\mathscr{B}^{\prime}$ as the set of bonds, equal to $K$ on $\mathscr{B}$ and to $K^{\prime}$ on $\mathscr{B}^{\prime}$.

Suppose now that both $\mathbb{L}$ and $\mathscr{B}$ are finite and let $K$ be an interaction with $\mathscr{B}$ as the set of bonds. The partition function is

$$
Z=\sum_{X \in \mathscr{X}} \exp \sum_{B \in \mathscr{B}} K(B) \sigma_{B}(X),
$$

and the so-called low temperature expansion $(L T E)$ for $Z$ reads

$$
Z=|\mathscr{S}| \prod_{B \in \mathscr{B}} e^{K(B)} \sum_{\beta \in \Gamma} \prod_{B \in \beta} e^{-2 K(B)} .
$$


Let

$$
Z^{0}=\sum_{\beta \in \Gamma} \prod_{B \in \beta} e^{-2 K(B)}
$$

and define a polynomial $M$ in the variables $z_{\mathscr{B}}=\left(z_{B}\right)_{B \in \mathscr{B}}$ by

$$
M\left(z_{\mathscr{B}}\right)=\sum_{\beta \in \Gamma} z^{\beta 9}
$$

where $z^{\beta}=\prod_{B \in \beta} z_{B}$. Then

$$
\left.M\left(z_{\mathscr{B}}\right)\right|_{z_{B}=\exp -2 K(B)}=Z^{0}(K) .
$$

3.2. Let $P$ be a complex polynomial in several variables which is of degree 1 with respect to each. That is there is a finite set $\mathscr{B}$ and

$$
P\left(z_{\mathscr{B}}\right)=\sum_{\beta \subset \mathscr{B}} c_{\beta} z^{\beta}
$$

Let $\mathscr{B}=\bigcup_{i} \mathscr{B}_{i}$ be a finite covering of $\mathscr{B}$ and let $P_{i}\left(z_{\mathscr{B}_{i}}\right)=\sum_{\beta C \mathscr{B}_{i}} c_{i, \beta} z^{\beta}$ be a family of polynomials. It is aid that $P\left(z_{\mathscr{B}}\right)$ is the Asano contraction of $\left(P_{i}\left(z_{\mathscr{B}_{i}}\right)\right)_{i}$ if

$$
c_{\beta}=\prod_{i} c_{i, \beta \cap \mathscr{B}_{i}}
$$

We will say that the variable $z_{B}$ is contracted if $B$ belongs to more than one of $\left(\mathscr{B}_{i}\right)_{i}$.

Theorem (Ruelle [21]). Let $P\left(z_{\mathscr{B}}\right)$ be the Asano contraction of $\left(P_{i}\left(z_{\mathscr{B}_{i}}\right)\right)$ and let for each $B \in \mathscr{B}_{i}$ a subset $R_{i, B}$ of the complex plane be given which is closed and does not contain 0 if $z_{B}$ is contracted.

Suppose that $P_{i}\left(z_{\mathscr{B}_{i}}\right)$ is nonzero if $z_{B} \notin R_{i, B}$, all $B \in \mathscr{B}_{i}$. Then $P\left(z_{\mathscr{B}}\right)$ does not vanish when $z_{B} \notin-\prod_{i}\left(-R_{i, B}\right)$ for all $B \in \mathscr{B}$; here for a finite sequence $\left(R_{i}\right)_{i=1}^{n}$ of subsets of $\mathbb{C}$

$$
\prod_{i=1}^{n} R_{i}=\left\{z_{1} \ldots z_{n}: z_{i} \in R_{i}, i=1, \ldots, n\right\}
$$

3.3. Proposition([13] Section 2.2). $M\left(z_{\mathscr{B}}\right)$ is the Asano contraction of $\left(M\left(z_{\mathscr{B}_{i}}\right)\right)$ if and only if the subgroup of $\mathscr{P}(\mathscr{B})$ generated by $\bigcup_{i} \mathscr{K}_{i}$ coincides with $\mathscr{K}$ (we write then: $\left.\left[\bigcup_{i} \mathscr{K}_{i}\right]=\mathscr{K}\right)$.

3.4. Let now $\mathbb{L}$ be a $v$-dimensional lattice in the sense of Sec. 1.4 and let $\mathscr{B}$ be a family of bonds as in Section 3.1. We assume that in addition to the mapping $B \mapsto \bar{B}$ there is defined another mapping $B \mapsto \operatorname{supp}(B)$ from $\mathscr{B}$ to $\mathscr{P}_{f}(\mathbb{L}) \backslash\{\emptyset\}$, and that always

$$
\bar{B} \subset \operatorname{supp}(B) \text {. }
$$

We also assume that $\mathscr{B}$ is a $\mathbb{Z}^{v}$-set and that the actions of $\mathbb{Z}^{v}$ on $\mathscr{B}$ and $\mathbb{L}$ commute with both $B \mapsto \bar{B}$ and $B \mapsto \operatorname{supp}(B)$; this setup will be of use in Section 4 (cf. Section 4.4 for definitions).

Suppose now that $\mathscr{B}$ is as above, that $K$ is a $\mathbb{Z}^{v}$-invariant interaction with bonds $\mathscr{B}$ and that for any $a \in \mathbb{L}$

$$
\sum_{\operatorname{supp}(B)_{\ni} a}|K(B)|<\infty \text {. }
$$

9 In contradistinction to [13, Section 2.2] here $M$ depends not only on the family $\mathscr{B}$ but also on the mapping $B \mapsto \bar{B}$. 
Then for a finite subset $\Lambda$ of $\mathbb{L}$ one defines

$$
\begin{aligned}
\mathscr{B}_{A} & =\{B \in \mathscr{B}: \operatorname{supp}(B) \subset A\}, \mathscr{K}_{A}=\mathscr{K}\left(\mathscr{B}_{A}\right) \\
Z_{A} & =\sum_{X \in \mathscr{X}_{A}} \exp \sum_{B \in \mathscr{B}_{A}} K(B) \sigma_{B}(X) \\
Z_{A}^{0} & =\sum_{\beta \in \Gamma\left(\mathscr{B}_{A}\right)} \prod_{B \in \beta} e^{-2 K(B)} .
\end{aligned}
$$

If $(\Lambda)$ is a Van Hove net then the limits

$$
\begin{aligned}
p=\lim _{\Lambda} \frac{1}{|\Lambda|} \log Z_{\Lambda}, p^{0} & =\lim _{\Lambda} \frac{1}{|\Lambda|} \log Z_{\Lambda}^{0} \\
s^{0} & =\lim _{\Lambda} \frac{1}{|\Lambda|} \log \left|\mathscr{S}_{\Lambda}\right|
\end{aligned}
$$

exist and

$$
p=p^{0}+s^{0}+\sum_{B \in \mathscr{B}_{0}} K(B)
$$

for any fundamental family of bonds $\mathscr{B}_{0}$.

3.5. Let now $\mathbb{L}$ and $\mathscr{B}$ be as in the preceding section and assume that $\mathscr{B}$ has a finite fundamental family. Let $\mathbb{L}_{0}$ be a fundamental subfamily of $\mathbb{L}$. For a subset $\Lambda$ of $\mathbb{Z}^{v}$ we set

$$
\Lambda^{\mathbb{L}_{0}}=\left\{a+x: a \in \mathbb{L}_{0}, x \in \Lambda\right\} .
$$

Theorem. There exists a parallelepiped $\Lambda_{0} \subset \mathbb{Z}^{v}$ such that for any large enough parallelepiped $\Lambda \subset \mathbb{Z}^{v}$

$$
\mathscr{K}_{\Lambda}^{\mathbb{L}_{0}}=\left[\bigcup_{i} \mathscr{K}_{\Lambda_{i}}^{\mathbb{L}_{0}}\right]
$$

where $\left\{\Lambda_{i}\right\}$ are the translates of $\Lambda_{0}$ contained in $\Lambda$.

This is a special case of Theorem $1, \S 5$ of [5]: Define a function $s: \mathscr{P}_{f}(\mathscr{B}) \rightarrow \mathbb{Z}^{v}$ by

$$
s(\beta)=\bigcup_{B \in \beta}\left\{x \in \mathbb{Z}^{v}: \operatorname{supp}\left(\tau_{-x}(B)\right) \text { intersects } \mathbb{L}_{0}\right\}
$$

and consider the natural action of $\mathbb{Z}^{v}$ on $\mathscr{P}_{f}(\mathscr{B})$ and on itself. Abelian groups can be always considered as $\mathbb{Z}$-modules, in our case even $\mathbb{Z}_{2}$-modules, and it is easy to see that since $\mathscr{B}_{0}$ is finite the $\mathbb{Z}_{2}\left[\mathbb{Z}^{v}\right]$-module $\mathscr{P}_{f}(\mathscr{B})$ is a finite $A[G]$ module, $A=\mathbb{Z}_{2}, G=\mathbb{Z}^{v}$, in the sense of [5]. Now to get the needed result we can apply Theorem $1, \S 5$ of [5] to $M=\mathscr{P}_{f}(\mathscr{B}), M_{0}=\mathscr{K}$ and the convenient family of $[5, \S 6]$.

3.6. The correlation functions will be investigated with help of Proposition 1.3. Therefore we need information on the change of the polynomials $M\left(z_{\mathscr{B}}\right)$ under suitable changes of the interaction, [13, Section 1.7]:

Suppose that $A_{1}, \ldots, A_{n} \in \overline{\mathscr{B}}$ and let $\mathscr{B}{ }^{\prime}=\mathscr{B} \cup\left\{A_{1}, \ldots, A_{n}\right\}$. Let $\alpha_{1}, \ldots, \alpha_{n} \in \mathscr{P}(\mathscr{B})$ be such that $\bar{\alpha}_{1}=A_{i}$ and let $\alpha_{i}^{\prime}=\alpha_{i} \cup\left\{A_{i}\right\}$. Then $\mathscr{K}\left(\mathscr{B}^{\prime}\right)$ is generated by $\mathscr{K}(\mathscr{B}) \cup$ $\left\{\alpha_{1}^{\prime}, \ldots, \alpha_{n}^{\prime}\right\}$.

The polynomials $M\left(z_{\alpha_{i}^{\prime}}\right)$ have especially simple structure when $\alpha_{i}$ are minimal subfamilies of $\mathscr{B}$ that yield $A_{i}$. Namely, consider a family $\alpha$ of bonds with the property that $\mathscr{K}(\alpha)$ is trivial; let $A=\bar{\alpha}$ and $\alpha^{\prime}=\alpha \cup\{A\}$. Then, [13, Sec. 2.3],

$$
M\left(z_{\alpha^{\prime}}\right)=\frac{1}{2}\left[\left(1+z_{A}\right) \prod_{B \in \alpha}\left(1+z_{B}\right)+\left(1-z_{A}\right) \prod_{B \in \alpha}\left(1-z_{B}\right)\right] .
$$


We will need the following property of $M\left(z_{\alpha^{\prime}}\right)$ :

There exists a function $\delta \rightarrow \varepsilon(\delta), \delta, \varepsilon>0$, such that $\left|z_{A}-1\right|<\delta$ and $\left|z_{B}+1\right|>\varepsilon(\delta)$, $B \in \alpha$, imply that $M\left(z_{\alpha^{\prime}}\right) \neq 0$.

This follows readily from (3.1) and can be sharpened as follows.

Let $D(\lambda)$ be the interior of the circle which is symmetric with respect to the real axis and is passing through points $(1-\lambda)(1+\lambda)^{-1}$ and $(1+\lambda)(1-\lambda)^{-1}$. Then $M\left(z_{\alpha^{\prime}}\right) \neq 0$ if $z_{A} \in D(\lambda)$ and $z_{B} \in-D\left(\lambda \frac{1}{|\alpha|}\right), 0<\lambda<1$.

3.7. If $|\alpha|=2$ then $M\left(z_{\alpha^{\prime}}\right) \neq 0$ for $z_{A}$ real, $0 \leqq z_{A}<1$, and $\left|z_{B}\right|<1, B \in \alpha$.

This can be shown straightforward; cf. also [17] Theorem 1.3, and Lemma 1.4.

\section{Analyticity and Uniqueness}

4.1. Reduced Pressure. Let IL be a $v$-dimensional lattice in the sense of Section 1.2 and let $\mathbb{L}_{0}$ be a fundamental subset of $\mathbb{L}$. Let $\mathscr{B}$ be the family of bonds of a spin $\frac{m}{2}$ system with an interaction $K$ and with a finite fundamental family $\mathscr{B}_{0}$. For a finite subset $\Lambda$ of $\mathbb{L}$ the partition function

$$
Z_{A}=\sum_{X \in \mathscr{X}_{A}} \exp \sum_{B \in \mathscr{B}_{A}} K(B) s_{B}(X)
$$

where $\mathscr{B}_{\Lambda}=\{B \in \mathscr{B}: \operatorname{supp} B \subset \Lambda\}$ will be written as

$$
Z_{A}=\left|\mathscr{S}_{\Lambda}\right|\left(\exp \sum_{B \in \mathscr{B}_{A}} m^{|B|} K(B)\right) Z_{A}^{0} ;
$$

here $\mathscr{S}_{A}$ is the symmetry group of the subsystem in $\Lambda$ defined as in (2.9).

It is not hard to see that the Van Hove limits

$$
s^{0}=\lim _{\Lambda} \frac{1}{|\Lambda|} \log \left|\mathscr{S}_{\Lambda}\right|, \quad \lim _{\Lambda} \frac{1}{|\Lambda|} \sum_{B \in \mathscr{B}_{\Lambda}} m^{|B|} K(B)
$$

exist, the second being equal to $\sum_{B \in \mathscr{B}_{0}} m^{|B|} K(B)$. Therefore also exists

$$
p^{0}=\lim _{\Lambda} \frac{1}{|\Lambda|} \log Z_{\Lambda}^{0}
$$

and

$$
p=p^{0}+s^{0}+\sum_{B \in \mathscr{B}_{0}} m^{|B|} K(B) ;
$$

$p^{0}$ will be called the reduced pressure.

4.2. To introduce the spin $\frac{1}{2}$ system on $\mathbb{I}^{m}$ corresponding to the system of the preceding section we define first the bonds. For each $B \in \mathscr{B}$ we need such a family $B^{m}$ of bonds (in the sense of Section 3.1) of the spin $\frac{1}{2}$ system that

$$
\prod_{a \in \operatorname{supp}(B)}\left(\sigma_{a, 1}+\ldots+\sigma_{a, m}\right)^{B(a)}=\sum_{C \in B^{m}} \sigma_{C} .
$$

Let us therefore introduce for each $(a, i, n) \in \mathbb{L} \times\{1, \ldots, m\} \times \mathbb{N}$ a variable $X_{a, i, n}$; let $B^{m}$ be the family of monomials of the polynomial

$\prod_{a \in \operatorname{supp}(B)} \prod_{n=1}^{B(a)}\left(X_{a, 1, n}+\ldots+X_{a, m, n}\right)$ 
and let

$$
\mathscr{B}^{m}=\bigcup_{B \in \mathscr{B}} B^{m} .
$$

The support is defined by

$$
\operatorname{supp}\left(\prod_{r} X_{a_{r}, i_{r}, n_{r}}\right)=\left\{\left(a_{r}, i_{r}\right)\right\}_{r},
$$

the action of $\mathbb{Z}^{v}$ on $\mathscr{B}^{m}$ by

$$
\tau_{x}\left(\prod_{r} X_{a_{r}, i_{r}, n_{r}}\right)=\prod_{r} X_{a_{r}+x, i_{r}, n_{r}}
$$

and $B \mapsto \bar{B}$ by

$$
\prod X_{a_{r}, i_{r}, n_{r}} \mapsto \sum_{r}\left\{\left(a_{r}, i_{r}\right)\right\}
$$

where $\sum_{r}$ means addition in the group $\mathscr{P}\left(\mathbb{L}^{m}\right)$. We define an interaction $K^{m}$ of spin $\frac{1}{2}$ system:

$$
K^{m}(C)=K(B) \quad \text { if } \quad C \in B^{m}
$$

and an interaction $R^{m}$ with $\{(a, i),(a, j): a \in \mathbb{L}, i, j=1, \ldots, m\}$ as the set of bonds and (1.5) as the coefficients.

4.3. The reduced pressures of the original and the spin $\frac{1}{2}$ systems are related as follows:

$$
p^{0}(K)=m p^{0}\left(K^{m}+R^{m}\right) .
$$

This follows from (1.4) and the following observations: $\mathscr{S}_{A}$ and $\mathscr{S}_{A^{m}}$ are of the same cardinality since, due to the interaction $R^{m}$, in every configuration of $\mathscr{S}_{A^{m}}$ the spins at $(a, r)$ and $(a, s)$ are aligned; $\left|B^{m}\right|=m^{|B|}$ and $\left|\Lambda^{m}\right|=m|\Lambda|$.

4.4. From now on we assume that $\bigcup_{B \in \mathscr{B}} \operatorname{supp}(B)=\mathbb{L}$. Otherwise, as is seen from the definition of equilibrium state, one can factor out the zero-interaction state on $\mathbb{L} \backslash \bigcup_{B \in \mathscr{B}} \operatorname{supp}(B)$.

We note that the bonds of $R^{m}$ are in $\overline{\mathscr{B}^{m}}$. More precisely, for any $(a, i) \in \mathbb{L}^{m}, i<m$, there exist $C_{1}, C_{2} \in \mathscr{B}^{m}$ such that

$$
\bar{C}_{1}+\bar{C}_{2}=\{(a, i),(a, i+1)\} .
$$

For, let $B \in \mathscr{B}$ be such that $a \in \operatorname{supp}(B)$ and consider, for instance,

$$
\begin{aligned}
& C_{1}=X_{a, i, 1} \prod_{n=2}^{B(a)} X_{a, 1, n} \prod_{\substack{b \in \operatorname{supp}(B) \\
b \neq a}} \prod_{n=1}^{B(b)} X_{b, 1, n} \\
& C_{2}=X_{a, i+1,1} \prod_{n=2}^{B(a)} X_{a, 1, n} \prod_{\substack{b \in \operatorname{supp}(B) \\
b \neq a}} \prod_{n=1}^{B(b)} X_{b, 1, n}
\end{aligned}
$$

then (5.3) holds.

We also note that if $A$ is such an m.f. that $\bar{A} \in \overline{\mathscr{B}}$ then $\overline{A^{m}} \subset \overline{\mathscr{B}}$.

For it is seen from the definitions of $A^{m}$ and $C \mapsto \bar{C}, C \in A^{m}$, that if $A=A_{2}+A_{1}$ (in the sense that $A(a)=A_{1}(a)+A_{2}(a)$, all $a \in \mathbb{L}$ ) then $\overline{A^{m}} C \overline{A_{1}^{m}}+\overline{A_{2}^{m}}$. If we now define $A_{1}(a)=1$ if $A(a)$ is odd and 0 otherwise, then $A_{2}(a)=A(a)-A_{1}(a)$ is an even, non-negative integer and $A=A_{1}+A_{2}$. Since, as noted above, $\{(a, i),(a k i+1)\} \in$ $\overline{\mathscr{B}^{m}}, \overline{A_{2}^{m}} \subset \overline{\mathscr{B}^{m}}$. It is also clear that $\overline{A_{1}^{m}} \subset \overline{\mathscr{B}^{m}}$. 
4.5. Theorem ${ }^{10}$. Let $\mathbb{L}, \mathscr{B}, \mathscr{B}_{0}$ be as in Section 4.1. Then there exist positive numbers $r_{B}, B \in \mathscr{B}_{0}$, such that

(a) there exists a function $f$ of complex variables $z_{\mathscr{B}_{0}}=\left(z_{B}\right)_{B \in \mathscr{B}_{0}}$, analytic in the polydisc $\left|z_{B}\right|<r_{B}$ such that for any interaction $K$ with bonds $\mathscr{B}$

$$
\left.f\left(z_{\mathscr{B}_{0}}\right)\right|_{Z_{B}=\exp -2 K(B)}=p^{0}(K)
$$

if $\exp -2 K(B)<r_{B}$;

(b) if $\exp -2 K(B)<r_{B}$ then for each $m . f$. A such that $\bar{A} \in \overline{\mathscr{B}}, \varrho\left(s_{A}\right)$ has the same value for all $\varrho \in \Delta_{K}^{I}$, and $\varrho\left(s_{A}\right)$ extend to an analytic function of the variables $z_{\mathscr{B}_{0}}$ as in (a) (for definitions of $\bar{A}$ and $\overline{\mathscr{B}}$ cf. (2.6) and following text).

We consider first the spin $\frac{1}{2}$ case.

Let $M^{\Lambda}\left(z_{\mathscr{B}_{0}}\right)$ be the polynomial in the variables $z_{\mathscr{B}_{0}}$ obtained from $M\left(z_{\mathscr{B}_{A}}\right)$ by substitution $z_{B}, B \in \mathscr{B}_{0}$, for each $z_{B^{\prime}}, B^{\prime} \in \mathscr{B}_{A}$, for which $B^{\prime}$ is congruent with $B$. Since

$$
\left.M^{\Lambda}\left(z_{\mathscr{B}_{0}}\right)\right|_{z_{B}=\exp -2 K(B)}=Z_{\Lambda}^{0}(K)
$$

and $M^{\Lambda}$ has non-negative coefficients, to be able to apply the Lee-Yang method ${ }^{11}$ it is enough to find a van Hove net $\Lambda_{\alpha}$ such that $M^{\Lambda} \alpha\left(z_{\mathscr{B}_{0}}\right) \neq 0$ if $\left|z_{B}\right|<r_{B}$. By Proposition 3.3 and Theorem 3.4, there exists a parallelepiped $\Lambda_{0} \subset \mathbb{Z}^{v}$ such that for each large enough parallelepiped $\Lambda$ in $\mathbb{Z}^{v}, M\left(z_{\mathscr{B}_{\Lambda} \mathbb{L}_{0}}\right)$ is the Asano contraction of $\left(M\left(z_{\mathscr{B}_{\Lambda_{i}}^{\mathrm{L}} \mathrm{o}}\right)\right)$, where $\left\{\Lambda_{i}\right\}$ are the translates of $\Lambda_{0}$ that are contained in $\Lambda . M\left(z_{\mathscr{B}_{\Lambda_{0}}^{\mathrm{I}} \mathrm{D}}\right)$ is a polynomial with free term equal to 1 . Therefore it is non-zero if all its arguments are small enough, say $\left|z_{B}\right|<r$. On the other hand each $B \in \mathscr{B}$ is contained in no more than $\left|\Lambda_{0}\right|$ of $\left\{\mathscr{B}_{\left.\Lambda_{l}^{\mathrm{L}_{0}}\right\}}\right\}$. Therefore, by Theorem 3.2, $M\left(z_{\mathscr{B}_{A}^{L}}^{\mathbb{L}_{0}}\right) \neq 0$ if $\left|z_{B}\right|<r^{\left|\Lambda_{0}\right|}, B \in$ $\mathscr{B}_{\Lambda}^{\mathbb{L}_{0}}$. This yields the required property of $M^{\Lambda^{\mathbb{L}_{0}}}\left(z_{\mathscr{B}_{0}}\right)$.

To prove (b), again in the spin $\frac{1}{2}$ case, we consider an interaction $K^{\prime}$ with one-element family $\{A\}$ of fundamental bonds and such that $\bar{A} \in \overline{\mathscr{B}}$. We fix $\alpha \in \mathscr{P}_{f}(\mathscr{B})$ such that $\bar{\alpha}=\bar{A}$ and such that no subset of $\alpha$ has this property. If $C \in \mathscr{B}\left(K^{\prime}\right)$ then by $\alpha_{C}$ we denote the translate of $\alpha$ that yields $\bar{C}$ and we set $\alpha_{C}^{\prime}=\alpha_{C} \cup\{C\}$. For $A \subset \mathbb{L}$, we set

$$
\left.\mathscr{B}_{\Lambda}^{\prime}=\mathscr{B}_{\Lambda} \cup\left\{C \in \mathscr{B}\left(K^{\prime}\right)\right\}: \alpha_{C} \subset \mathscr{B}_{\Lambda}\right\} .
$$

Since $\mathscr{K}\left(\mathscr{B}_{A}^{\prime}\right)$ is generated by $\mathscr{K}\left(\mathscr{B}_{A}\right)$ and $\left\{\alpha_{C}^{\prime}: \alpha_{C} \subset \mathscr{B}_{A}\right\}$ (Section 3.5), $M\left(z_{\mathscr{B}_{A}^{\prime}}\right)$ is the Asano contraction of $M\left(z_{\mathscr{B}_{A}}\right)$ and $\left(M\left(z_{\alpha^{\prime} C}\right)\right)_{\alpha_{C} \subset \mathscr{B}_{A}}$ (Proposition 33). On the other hand, for each $\delta>0$ there exists $\varepsilon(\delta)$ such that $\left|z_{C}-1\right|<\delta$ and $\left|z_{B}+1\right|>\varepsilon$, $B \in \alpha_{C}$, implies that $M\left(z_{\alpha_{C}^{\prime}}\right) \neq 0$, all $C \in \mathscr{B}\left(K^{\prime}\right)$ (Section 3.6). The variables $z_{C}$ are not contracted in passing from $M\left(z_{\mathscr{B}_{A}}\right)$ and $\left(M\left(z_{\alpha_{C}^{\prime}}\right)\right)_{C}$ to $M\left(z_{\mathscr{B}_{A}^{\prime}}\right)$. Therefore, by Theorem 3.2, if $M\left(z_{\mathscr{B}_{A}}\right) \neq 0$ for $\left|z_{B}\right|<r_{B}$ then $M\left(z_{\mathscr{B}_{A}^{\prime}}\right) \neq 0$ for $\left|z_{B}\right|<r_{B} \cdot(1-\varepsilon)^{\left|\alpha_{C}\right|}$ and $\left|z_{C}-1\right|<\delta$; here the power $\left|\alpha_{C}\right|$ is due to the fact that a bond of $\mathscr{B}$ can appear in at most $\left|\alpha_{C}\right|$ of $\alpha_{C}$ 's.

10 Cf. [13] in the case of spin $\frac{1}{2}$ regular systems in $\mathbb{Z}^{\nu}$. Ref. [20] containes a remark that the results of [13] refer only to systems with even bonds. This, and some of the claims of generalizations, seems to result from overlooking Section 2.5 of [13] and of the need of a result like Theorem 3.5.

11 See [11, p. 111] (also [13, Section 2.4]). The multivariable version of the Vitali theorem can be found in [18]. We don't have a reference for corresponding generalization of the Hurwitz's theorem but it is not hard to see that it holds. 
Let us now take $\Lambda$ of the type $P^{\mathbb{L}_{0}}$ where $P$ is a large enough parallelepiped in $\mathbb{Z}^{v}$. By the first part of the proof $M\left(z_{\mathscr{B}_{A}}\right) \neq 0$ if $\left|z_{B}\right|<r_{B}\left(r_{B}=r^{\left|\Lambda_{0}\right|}\right.$ there) and, by the argument above, the polynomial $M^{\Lambda}\left(z_{\mathscr{B}_{0}^{\prime}}\right)$ if the variables $z_{\mathscr{B}_{0}^{\prime}}=\left(z_{\mathscr{B}_{0}^{\prime}} z_{A}\right)$ obtained from $M\left(z_{\mathscr{B}_{A}^{\prime}}\right)$ as $M^{\Lambda}\left(z_{\mathscr{B}_{0}}\right)$ from $M\left(z_{\mathscr{B}_{A}}\right)$ is non-zero if $\left|z_{B}\right|<r_{B} \cdot(1-\varepsilon)^{\left|\alpha_{A}\right|}, B \in \mathscr{B}_{0}$, $\left|z_{A}-1\right|<\delta$.

It is clear that

$$
\left.M^{\Lambda}\left(z_{\mathscr{B}_{0}}\right)\right|_{z_{B}=\exp -2 K(B), z_{A}=\exp -2 K^{\prime}(A)} \text {. }
$$

differs from $Z_{\Lambda}^{0}\left(K+K^{\prime}\right)$ in a way that does not affect the thermodynamic limit. Therefore we conclude that for each $\delta>0$ there exists $\varepsilon(\delta)>0, \varepsilon(\delta) \rightarrow 0$ as $\delta \rightarrow 0$, and a function $f_{\delta}$ of the complex variables $z_{\mathscr{B}_{0}^{\prime}}$ analytic in the polydisc $\left|z_{B}\right|<r_{B} \cdot(1-\varepsilon)^{\left|\alpha_{A}\right|}, B \in \mathscr{B}_{0},\left|z_{A}-1\right|<\varepsilon$ and such that

$$
\left.f_{\delta}\left(z_{\mathscr{B}_{0}^{\prime}}\right)\right|_{z_{B}=\exp -2 K(B), z_{A}=\exp -2 K^{\prime}(A)}=p^{0}\left(K+K^{\prime}\right) .
$$

Now Proposition 1.3 shows that $(b)$ holds in the regions exp $-2 K(B)<r_{B}$. $(1-\varepsilon(\delta))^{\left|\alpha_{A}\right|}, \delta$ varied. Letting $\delta \rightarrow 0$ concludes the proof in the spin $\frac{1}{2}$ case.

The above statements about the existence and properties of $f_{\delta}$ remain true, with obvious modifications, in case $K^{\prime}$ is any interaction with a finite fundamental family of bonds and such that $\overline{\mathscr{B}\left(K^{\prime}\right)} \subset \overline{\mathscr{B}(K)}$. Hence the $(a)$-part of the theorem for higher spins can be proved by reference to (4.2) and by noting that, because of (4.3) and Section 3.7, the polydisc of analyticity of $p^{0}\left(K^{m}+R^{m}\right)$ in the variables $z_{\mathscr{B}_{0}\left(K^{m}\right)}, R^{m}$ real, is the same as the one of $p^{0}\left(K^{m}\right)$. The method of the proof of $b$ ) in the spin $\frac{1}{2}$ case when applied to $p^{0}\left(K^{m}+R^{m}+\lambda K_{A}^{m}\right)$ yields $(b)$ for general spin; one has to use the fact that $\overline{A^{m}} \dot{\bar{C}} \overline{\mathscr{B}^{m}}$ (Section 4.2).

4.6. Corollary. For any ferromagnetic, finite range interaction system on a lattice the entropy is the same for all translation invariant equilibrium states at low enough temperature and has $s^{0}(c f .(4.1))$ as the zero-temperature limit.

This can be proved as Proposition 2.6 of [13].

4.7. Remarks. (a) If $\mathbb{I L}=\mathbb{Z}^{v}$ then $s^{0}=\log 2$ when $\overline{\mathscr{B}}$ is trivial and $s^{0}=0$ otherwise. In general $0 \leqq s^{0} \leqq \log 2$. It seems that is always a multiple of $\left|\mathbb{L}_{0}\right|^{-1} \times \log 2$.

(b) After [13] was published, I came across the paper [9] by Leff where the entropy of ferromagnetic spin $\frac{1}{2}$ systems at low temperatures is discussed. In [9] it is not assumed that the interaction is of a finite range; the assumption that nearest neighbours interact is not essential: the one-dimensional Ising system used for estimates in [9] can be replaced by trivial systems in the sense of [13, Section 3.5]. However, in [9] a different definition of the entropy is employed making hard a comparison with Corollary 4.6.

(c) It can be shown that $s^{0}$ is the entropy of the state defined as follows: it is the image of the Haar measure on $\mathscr{S}$ under the mapping $\mathscr{S} \rightarrow \mathscr{X}$ defined by

$$
\left(X_{a}\right)_{a \in \mathbb{L}} \rightarrow\left(m \cdot X_{a}\right)_{a \in \mathbb{L}} \in \mathscr{X} .
$$

In case of spin $\frac{1}{2}$ systems $\mathscr{S} \rightarrow \mathscr{X}$ is the inclusion mapping.

(d) From (2.11) and Theorem 4.5 one deduces the following about the zerotemperature limit of invariant equilibrium states:

The limit points of invariant equilibrium states as $T \rightarrow 0$ are $\mathscr{S}^{+}$-invariant measures on $\mathscr{X}$ concentrated (in the spin $\frac{1}{2}$ case) on $\mathscr{S}$. For example, $\lim _{T \rightarrow 0} \overline{\varrho^{+}}$is the 
Haar measure of $\mathscr{S}^{+}$, and $\lim _{T \rightarrow 0} \overline{\varrho^{+}}$is the Haar measure of $\mathscr{S}$. Here implicit is the fact that for low enough temperatures the group $\mathscr{S}^{+}$is temperature independent [cf. Section 7.6(b)]. For higher spin systems one has to consider the image measures in $\mathscr{X}$ as in (c).

4.8. We now summarize the results obtained so far. Combining Theorems 4.5 and 2.3 we get the following picture.

For any ferromagnetic, finite range interaction the invariant equilibrium states are at low enough temperature described by $\mathbb{Z}^{v}$-invariant measures on the compact group $\mathscr{S} / \mathscr{S}^{+}$with ergodic states corresponding to ergodic measures. Since $\mathscr{S} / \mathscr{S}^{+}$is the dual group of $\overline{\mathscr{B}} / \overline{\mathscr{B}}$ (Section 2.4 ) and $\overline{\mathscr{B}}$ is temperature independent and defined directly in terms of the interaction the problem is in a sense reduced to finding $\overline{\mathscr{B}^{+}}$. This is discussed in the next section.

\section{Examples. Systems in $\mathbb{Z}^{v}$}

5.1. Suppose that the ferromagnetic finite range interaction on a lattice $\mathbb{L}$ has only even bonds, as in the case of

$$
-\sum J_{a b} s_{a}^{2} s_{b}^{2}, \quad J_{a b}>0
$$

Then $\overline{\mathscr{B}}$ is trivial and therefore $\mathscr{S}=\mathscr{G}$. It is also not hard to see, directly from (1.2), that any equilibrium state is $\mathscr{G}$-invariant. This yields $\mathscr{S}=\mathscr{S}^{+}$and the conclusion that for such systems there is only one invariant equilibrium state for low enough temperatures.

In the case of spin 1 systems with interaction (6.1) the invariant equilibrium state is unique at all temperatures: the energy when written in terms of spin $\frac{1}{2}$ variables $\sigma_{a, 1}, \sigma_{a, 2}$ takes the form

$$
\begin{aligned}
\sum_{a, b} J_{a, b} s_{a}^{2} s_{b}^{2} & =\sum_{a, b} J_{a, b}\left(2+2 \sigma_{a, 1} \sigma_{a, 2}\right)\left(2+2 \sigma_{b, 1}, \sigma_{b, 2}\right) \\
& =4 \sum_{a, b}\left(J_{a, b}+J_{a, b} \tau_{a}+J_{a, b} \tau_{b}+J_{a, b} \tau_{a} \tau_{b}\right)
\end{aligned}
$$

where $\tau_{a}=\sigma_{a, 1} \sigma_{a, 2} \tau_{b}=\sigma_{b, 1} \sigma_{b, 2}$; i.e. it has the same structure as ferromagnetic, spin $\frac{1}{2}$, two-body interaction with an external field. This remark can be developed into a proof (cf. the proof of Theorem 4.5).

5.2. A slightly larger family than that of Section 5.1 is formed by trivial systems. These are systems for which $\overline{\mathscr{B}}$ is generated by translates of one of its elements.

In this case the invariant equilibrium state is again unique at low temperatures. In case of spin $\frac{1}{2}$ systems one has uniqueness for all temperaturs [13].

5.3. If $\mathbb{L}$ splits into more than one $\mathscr{B}$-component [13, Section 4.9]:

$$
\mathbb{L}=\bigcup_{i} \mathbb{L}_{i}
$$

and $\mathscr{S}_{i}, \mathscr{S}_{i}^{+}$are the corresponding groups then

$$
\mathscr{S} / \mathscr{S}^{+}=\prod_{i} \mathscr{S}_{i} / \mathscr{S}_{i}^{+}
$$

and the action of $\mathbb{Z}^{v}$ on $\mathscr{S} / \mathscr{S}^{+}$is reduced to the action on $\left\{\mathbb{L}_{i}\right\}$ and $\mathscr{S}_{i} / \mathscr{S}_{i}^{+}$. 


\subsection{Interactions of the Type}

$-\sum_{a, b} J_{a, b} S_{a} S_{b}, \quad J_{a b}>0$

on $\mathbb{Z}^{v}$. By Section 5.3 it is enough to consider the case when $\mathbb{Z}^{v}$ is connected with respect to the interaction. Then [13, Section 4.9] $\overline{\mathscr{B}}$ is the family of all finite, even subsets of $\mathbb{Z}^{v}$ and hence $\mathscr{S}$ has just two elements $E, F$ :

$$
E_{a}=1, F_{a}=-1 \text {, all } a \in \mathbb{Z}^{v} \text {. }
$$

On the other hand, by Section 5.6, $\overline{\mathscr{B}^{+}}=\mathscr{P}_{f}\left(\mathbb{Z}^{v}\right)$ which implies that $\mathscr{S}^{+}=\{E\}$. Thus $\mathscr{S} / \mathscr{S}^{+}$has two elements, both of them invariant under $\mathbb{Z}^{v}$. It follows that there are exactly two ergodic states at low enough temperatures.

The same holds for interaction of the type

$-\sum_{a, b, A} J(a, b, A) s_{a} s_{b} s_{A}-\sum_{B} J(B), \quad J>0$

with even m.f.'s $A, B$, provided $\mathbb{Z}^{v}$ is connected with respect to the family

$\{\{a, b\}:$ there exists $A$ such that $J(a, b, A) \neq 0\}$.

5.5. Ferromagnetic interaction of the type

$-\sum_{A} J(A) s_{A}-\sum_{a} J_{a} s_{a}$

leads to a unique invariant equilibrium state at low temperatures since in this case $\overline{\mathscr{B}}=\mathscr{P}_{f}(\mathbb{L})$. Similarly for any ferromagnetic interaction with $\overline{\mathscr{B}}=\mathscr{P}_{f}(\mathbb{L})$, as for instance

$\sum_{a, b} J(a, b) s_{a} s_{b}-\sum_{a, b, c} J(a, b, c) s_{a} s_{b} s_{c}$

5.6. The inequalities (1.9) and (1.8) imply that for a system of arbitrary spin $\overline{\mathscr{B}^{+}}$at low temperatures is the same as for spin $\frac{1}{2}$ system with bonds

$\{\bar{B}: B \in \mathscr{B}\}$.

This allows the results of [6] to extend as described below; for completeness we repeat the definitions from [6].

One defines in the abelian group $\mathscr{P}_{f}\left(\mathbb{Z}^{v}\right)$ a ring structure by setting

$$
A \cdot B=\sum_{a \in A, b \in B}\{a+b\} \quad A, B \in \mathscr{P}_{f}\left(\mathbb{Z}^{v}\right) ;
$$

the sum $\sum$ is taken here in $\mathscr{P}_{f}\left(\mathbb{Z}^{v}\right)$ and $a+b$ in $\mathbb{Z}^{v}$. The mapping

$A \mapsto$ characteristic function of $A$

gives an isomorphism of this ring, in fact an algebra over the field $\mathbb{F}_{2}=\{0,1\}$, with the group algebra $\mathbb{F}_{2}\left[\mathbb{Z}^{v}\right]$. We write $\mathbb{F}_{2}\left[\mathbb{Z}^{\nu}\right]$ instead of $\mathscr{P}_{f}\left(\mathbb{Z}^{v}\right)$ when considering this ring structure.

Let $X_{1}, \ldots, X_{v}$ be the elements of $\mathbb{F}_{2}\left[Z^{v}\right]$ corresponding to the elements of the natural basis of $\mathbb{Z}^{v}$ :

$$
X_{i}=\left\{\left(\delta_{i j}\right)_{j=1}^{v}\right\} .
$$

For $a \in \mathbb{Z}^{v}$ we set

$$
X^{a}=X_{1}^{a_{1}} \ldots X_{v}^{a_{v}} .
$$


Each element of $\mathbb{F}_{2}\left[\mathbb{Z}^{v}\right]$ can be written as

$$
\sum_{a \in A} X^{a}
$$

and, under the above identification of $\mathbb{F}_{2}\left[\mathbb{Z}^{v}\right]$ and $\mathscr{P}_{f}\left(\mathbb{Z}^{v}\right),(6.2)$ is identified with $A$. As is easy to see, $X^{a}, a \in \mathbb{Z}^{v}$, are the only invertible elements (units) of $\mathbb{F}_{2}\left[\mathbb{Z}^{v}\right]$; multiplication by $X^{a}$ leads to the translation by $a$ of the corresponding subset of $\mathbb{Z}^{v}$. The subalgebra of $\mathbb{F}_{2}\left[\mathbb{Z}^{v}\right]$ generated by $X_{1}, \ldots, X_{v}$ is isomorphic to the algebra $\mathbb{F}_{2}\left[X_{1}, \ldots, X_{v}\right]$ of polynomials in $v$ variables over $\mathbb{F}_{2}$. Since each element of $\mathbb{F}_{2}\left[X_{1}, \ldots, X_{v}\right]$ admits unique facorization into prime elements and each element of $\mathbb{F}_{2}\left[\mathbb{Z}^{v}\right]$ after multiplication by a unit is in $\mathbb{F}_{2}\left[X_{1}, \ldots, X_{v}\right], \mathbb{F}_{2}\left[\mathbb{Z}^{\nu}\right]$ has also the unique factorization property. It follows that for any family of elements of $\mathbb{F}_{2}\left[\mathbb{Z}^{v}\right]$ there is a unique up to a $X^{a}$-factor greatest common divisor.

An element $A$ of $\mathbb{F}_{2}\left[\mathbb{Z}^{v}\right]$ will be called regular if there exist $a, a_{1}, \ldots, a_{v} \in \mathbb{Z}^{v}$ and polynomials $P_{1}, \ldots, P_{v}$ in one variable such that

$$
A=X^{a} P_{1}\left(X^{a_{1}}\right) \ldots P_{v}\left(X^{a_{v}}\right),
$$

and $X^{a_{1}}, \ldots X^{a_{v}}$ (together with their inverses) generate $\mathbb{F}_{2}\left[\mathbb{Z}^{\nu}\right]$. If $a_{i}=\left\{\left(\delta_{i j}\right)_{j=1}^{\nu}\right\}$ this amounts to saying that $A$ factorizes into cartesian product of subsets of $\mathbb{Z}$. $\mathscr{B}$, or the system, is called regular if $\overline{\mathscr{B}}$ contains a regular element.

Theorem. Suppose that $\mathscr{B}$ is regular. Then for low enough temperatures $\overline{\mathscr{B}}{ }^{+}$is the principal ideal $(D)$ of $\mathbb{F}_{2}\left[Z^{\nu}\right]$ generated by a greatest common divisor (g.c.d.) $D$ of $\{\bar{B}: B \in \mathscr{B}\}$.

In other words, $\overline{\mathscr{B}^{+}}$is the smallest $\mathbb{Z}^{v}$-invariant subgroup of $\mathscr{P}_{f}\left(\mathbb{Z}^{v}\right)$ that contains $D$. As in [6], it is natural to conjecture that the theorem holds without the regularity assumption. We illustrate the theorem by showing that in the example of Section $5.4 \overline{\mathscr{B}} \overline{\mathscr{P}}_{f}\left(\mathbb{Z}^{v}\right)$ at low enough temperatures, and by applying it in Section 5.8; further examples and a discussion can be found in [6].

Suppose that $\overline{\mathscr{B}}$ consists of all finite, even subsets of $\mathbb{Z}^{v}$. Then $\overline{\mathscr{B}}$ contains $1+X_{1}, \ldots, 1+X_{v}$. It follows that the interaction is regular and that $D=1$ is a g.c.d. By Theorem $\overline{\mathscr{B}^{+}}=\mathbb{F}_{2}\left[\mathbb{Z}^{v}\right] \cdot 1=\mathbb{F}_{2}\left[\mathbb{Z}^{v}\right]\left(=\mathscr{P}_{f}\left(\mathbb{Z}^{v}\right)\right)$.

5.7. If, in the setup of the preceding section, $D$ is a g.c.d. for $\overline{\mathscr{B}}$, then, as far as the problem of determination of $\mathscr{S} / \mathscr{S}^{+}$is concerned, we can in the spin $\frac{1}{2}$ case pass to a system with bonds $\mathscr{B}^{\prime}$ :

$$
\mathscr{B}=\left\{B^{\prime} \cdot D: B^{\prime} \in \mathscr{B}^{\prime}\right\} \text {. }
$$

After this reduction

$$
\overline{\mathscr{B}^{\prime+}}=\mathscr{P}_{f}\left(\mathbb{Z}^{v}\right), \mathscr{S}^{\prime+}=\{0\}
$$

and, see [6, Remark],

$$
\mathscr{S} / \mathscr{S}^{+} \cong \mathscr{S}^{\prime} \text {. }
$$

Therefore, the following yields a criterion of the finiteness of the number of ergodic equilibrium states at low temperatures in general.

$\mathscr{S}$ is finite if and only if there exist $n_{1}, \ldots, n_{v} \in \mathbb{Z}$ such that $\left\{0, n_{1} e_{1}\right\}, \ldots,\left\{0, n_{v} e_{v}\right\} \in \overline{\mathscr{B}}$. (Here $e_{1}, \ldots, e_{v}$ can be replaced by any family of $v$ linearly independent elements of $\mathbb{Z}^{v}$.) 
Finiteness of $\mathscr{S}$ is equivalent to the finiteness of $\mathscr{P}_{f}\left(\mathbb{Z}^{v}\right) / \overline{\mathscr{B}}$. If $\left\{0, n_{1} e_{1}\right\}, \ldots$, $\left\{0, n_{v} e_{v}\right\} \in \overline{\mathscr{B}}$ then, obviously, each element of $\mathscr{P}_{f}\left(\mathbb{Z}^{v}\right)$ is congruent mod $\mathscr{\mathscr { B }}$ with a subset of $\left[0,\left(n_{1}-1\right) e_{1}\right] \times \ldots x\left[0,\left(n_{v}-1\right) e_{v}\right]$; this shows that our condition is sufficient. Suppose now that $\mathscr{P}_{f}\left(Z^{v}\right) / \overline{\mathscr{B}}$ is finite. Then there exist a finite subfamily, say $\mathscr{A}$, of $\mathscr{P}_{f}\left(\mathbb{Z}^{v}\right)$ with the property that for any $C \in \mathscr{P}_{f}\left(Z^{v}\right)$ there exists $A \in \mathscr{A}$ such that $C+A \in \overline{\mathscr{B}}$. Let therefore $A_{n} \in \mathscr{A}$ be such that $\left\{n e_{1}\right\}+A_{n} \in \overline{\mathscr{B}}, n \in \mathbb{Z}$. Since $\mathscr{A}$ is finite $A_{n^{\prime}}=A_{n^{\prime \prime}}$ for some $n^{\prime} \neq n^{\prime \prime}$. It follows that $\left\{n^{\prime} e_{1}, n^{\prime \prime} e_{1}\right\} \in \overline{\mathscr{B}}$ and, by invariance under translations, also $\left\{0,\left(n^{\prime}-n^{\prime \prime}\right) e_{1}\right\} \in \overline{\mathscr{B}}$. Repeating the same with $e_{2}, \ldots, e_{v}$ we get that our condition is also a necessary one.

An alternative formulation of the criterion: $\mathscr{S}$ is finite iff $\overline{\mathscr{B}}$ contains a set of the form $\left\{(0, a)\right.$ : a running through a $v$-dimensional sublattice of $\left.\mathbb{Z}^{v}\right\}$.

It can be shown that if, in a reduced case, the above condition is not satisfied then the system admits infinitely many ergodic states at low enough temperature.

5.8. In this section we consider in some detail factorizable models: models on $\mathbb{Z}^{v}$ such that $\overline{\mathscr{B}}$ is generated by polynomials in one variable. For any factorizable model there exist $P_{1}, \ldots, P_{v} \in \mathbb{F}_{2}[\mathbb{Z}]$ such that the ideal $\overline{\mathscr{B}}$ of $\mathbb{F}_{i}\left[\mathbb{Z}^{v}\right]$ is generated by $P_{1}\left(X_{1}\right), \ldots, P_{v}\left(X_{v}\right)$. The Ising model in $v$ dimensions is of this type with $P_{i}(X)=$ $1+X, i=1, \ldots, v$.

It is plain that if at least two of $\left\{P_{i}\right\}$ are non-zero then the g.c.d. is a unit. Therefore by Theorem 5.6, in such a case $\overline{\mathscr{B}}{ }^{+}=\mathscr{P}_{f}\left(\mathbb{Z}^{\nu}\right)$ and hence $\mathscr{S} / \mathscr{S}^{+} \cong \mathscr{S}$. Moreover, if some of $\left\{P_{i}\right\}$ vanish then (cf. Section 5.3) the problem reduces to a similar one in a lower dimension with all of the polynomials non-zero, and it is not hard to see that one has continuously many ergodic equilibrium states at low tempreatures, cf. [14]. If all of the polynomials are zero, or only one of them is non-vanishing, we are in a situation described in Section 5.1 and 5.2 with unique invariant equilibrium state at low temperatures.

We identify restrictions of the configurations to straight lines parallel to the coordinate axes with the configurations of one-dimensional systems. We then note that $X \in \mathscr{S}\left(P_{1}, \ldots, P_{v}\right)$ if and only if for each line parallel to $i$-th axis the restriction is in $\mathscr{S}\left(P_{i}\right), i=1, \ldots, v$. We therefore begin with the case of $v=1$; we write $P(X)$ instead of $P_{1}\left(X_{1}\right)$ and $B$ for the corresponding bond.

$$
\begin{aligned}
& \left(X_{a}\right)_{a \in Z} \text { is in } \mathscr{S}(P) \text { if and only if } \\
& \prod_{b \in B} X_{a+b}=1, \forall a \in Z
\end{aligned}
$$

If $P=0$, i.e. $B=\emptyset$ then $\mathscr{S}=\mathscr{X}$. Otherwise we can choose $P=\sum_{b \in B} X^{b}$ to be

$$
1+a_{1} X+\ldots+a_{p-1} X^{p-1}+X^{p}, a_{i}=0,1,
$$

and it is clear that the coordinates $X_{a}, a=1, \ldots, p$, of $X \in \mathscr{S}$ can be chosen arbitrarily with all the other determined by Condition (6.3); we could identify the group $\mathscr{S}$ with $\mathscr{X}_{\{1, \ldots, p\}}$. It follows in particular that Card $(\mathscr{S})=2^{p}$. The action of $\mathbb{Z}$ on $\mathscr{S}(P)$ depends on $P$ in a more subtle way than the cardinality of $\mathscr{S}$, as the examples below demonstrate.

Let $P(X)=1+X+X^{2}+\ldots+X^{p}$. Then, as follows from (6.2) $X=\left(X_{a}\right) \in \mathscr{S}$ iff it is periodic with period $p+1$, i.e.

$$
X_{a+p+1}=X_{a}, \quad \forall a \in \mathbb{Z},
$$


and $\mathbb{Z}$ acts on $\mathscr{S}$ as $\mathbb{Z}_{p+1}{ }^{12}$ If $p+1$ is a prime member, as in the case of $1+X+X^{2}+$ $X^{3}+X^{4}$, there are no nontrivial subgroups of $\mathbb{Z}_{p+1}$ and hence the orbits of $\mathbb{Z}$ have either 1 or $p+1$ elements. Since, as is not hard to see, for $p$ prime $E\left(E_{a}=1, \forall a\right)$ is the only $\mathbb{Z}$-invariant element of $\mathscr{S}$ there are exactly

$$
1+\left(2^{p}-1\right) /(p+1)
$$

orbits in this case.

In a similar way, if $P(X)=1+X^{p}, p$ prime, one can see that there are

$$
2+\left(2^{p}-2\right) / p
$$

orbits, two one-element and the rest of $p$ elements.

For $P(X)=1+X+X^{4}$ one can see by inspection that there is an element $X$ of $\mathscr{S}$ containing the interval

$----+++-++--+-+----$

It follows that the orbit of $X$ is of fifteen elements. Since Card $(\mathscr{S})=2^{4}=16$ there are only two orbits: $\{E\}$, Orbit $(X)$.

Similarly, there are only two orbits in the case of $P(X)=1+X+X^{3}$. This can be seen by drawing any element of $\mathscr{S}$ different from $E$. We will deduce it from the following fact, proof of which is omitted:

If $\mathbb{Z}$ acts on $\mathscr{S}$ as $\mathbb{Z}_{n}$ and if $P$ is a prime polynomial then $n$ is a divisor of $2^{p}-1$.

To apply this to our polynomial we observe that were it not prime it would have $1+X$ as a factor, which is impossible since $P(1) \neq 0$. Therefore, $n$ is a divisor of $2^{3}-1=7$ and hence $n=7$. Since Card $(\mathscr{S})=2^{3}=8$, there are only two orbits: $\{E\}$ and one of seven elements. In the same way we prove that there are only two orbits for such prime $P$ for which $2^{p}-1$ is prime, as for instance in the case of $P(X)=1+X+X^{7}$.

We now pass to the general case and we consider a system in $\mathbb{Z}^{v}$ with none of $P_{1}, \ldots, P_{v}$ equal to zero. Let all the polynomials be chosen in the form (6.4), and let $p_{i}$ be the degree of $P_{i}, i=1, \ldots, v$. We first note that, as is not hard to see, if $\mathbb{Z}$ acts on $\mathscr{S}\left(P_{i}\right)$ as $\mathbb{Z}_{n_{i}}$ then $\mathbb{Z}^{v}$ acts on $\mathscr{S}\left(P_{1} \ldots, P_{v}\right)$ as $Z_{n_{1}} \times \ldots \times Z_{n_{v}}$. The discussion of the number of elements of $\mathscr{S}(P)$ shows that $\mathscr{S}\left(P_{1}, \ldots, P_{v}\right)$ is of $2^{p_{1} \ldots p_{v}}$ elements; this also gives a majorization of the number of ergodic equilibrium states at low temperatures. For some models more precise information is deduced from our discussion of one-dimensional systems:

1. Let $P_{2}=\ldots=P_{v}=1+X$. Then $\mathscr{S}\left(P_{1}, \ldots P_{v}\right)$ can be identified with $\mathscr{S}\left(P_{1}\right)$, with $\mathbb{Z}^{v}$ acting on $\mathscr{S}$ as $\mathbb{Z}_{n_{1}}$. For $P_{1}=1+X+X^{2}+\ldots+X^{p}, p+1$ prime, we are getting exactly

$$
1+\left(2^{p}-1\right) /(p+1)
$$

ergodic equilibrium states at low temperatures, one mixing $\left(\varrho^{+}\right)$and the others with $(p+1)$-element supports in $\mathscr{E}(\Delta)$.

2. $P_{2}, \ldots, P_{v}$ as above, $P_{1}=1+X^{p}, p$ prime, yield $2+\left(2^{p}-2\right) / p$ ergodic equilibrium states at low temperatures, two of them $\left(\varrho^{+}\right.$and $\left.\varrho^{-}\right)$mixing. The rest have $p$ extremal equilibrium states in the decomposition.

12 We say that $\mathbb{Z}^{v}$ acts on $\mathscr{S}$ as $G$ if $G$ is the quotient group of $\mathbb{Z}^{v}$ by the subgroup of these elements of $\mathbb{Z}^{v}$ that act trivially on $\mathscr{S}$. 
3. $P_{2}, \ldots, P_{v}$ as above, $P_{1}=1+X+X^{4}$; two ergodic equilibrium states at low temperatures, one mixing, the other one decomposing into a combination of fifteen extremals.

4. $P_{2}, \ldots, P_{v}$ as above, $P_{1}=1+X+X^{3}$ or $P_{1}(X)=1+X+X^{7}$, again two ergodic equilibrium states at low temperatures.

5. $v=2, P_{1}(X)=1+X+X^{2}+\ldots+X^{p}, P_{2}=1+X+X^{2}+\ldots+X^{q}$, with $p+1$ and $q+1$ prime; similiarly we could consider the case of $P_{i}(X)=1+X+X^{2}+\ldots+$ $X^{p_{i}}, i=1, \ldots, v$.

If $p \neq q$ the only nontrivial subgroups of $\mathbb{Z}_{p+1} \times \mathbb{Z}_{q+1}$ are $\mathbb{Z}_{p+1} \times\{0\}$ and $\{0\} \times \mathbb{Z}_{p+1}$. On the other hand, it is not hard to see that $E$ is the only $\{0\} \times \mathbb{Z}_{q+1}-$ or $\mathbb{Z}_{p+1} \times\{0\}-$ invariant element of $\mathscr{S}$. It follows that there are

$$
1+\left(2^{p q}-1\right) /(p+1)(q+1)
$$

ergodic equilibrium states at low temperatures, one mixing $\left(\varrho^{+}\right)$and the rest with $(p+1)(q+1)-$ element supports in $\mathscr{E}(\Delta)$.

If $p=q$ there are altogether

$$
1+p\left(2^{p}-1\right) /(p+1)+\left[2^{p^{2}}-p\left(2^{p}-1\right)-1\right] /(p+1)^{2}
$$

orbits of $\mathbb{Z}^{2}$ in $\mathscr{S}$, and therefore as much ergodic equilibrium states at low temperatures. Of these one is mixing, $p\left(2^{p}-1\right) /(p+1)$ have $(p+1)-$ element supports in $\mathscr{E}(\Delta)$ and the rest is of $(p+1)$ - element supports ${ }^{13}$.

One can combine the other one-dimensional examples in a similar way.

\section{Remarks}

6.1. The description of $\Delta^{+}$given in Theorem 2.3 , and presumably also Theorem 5.6, generalizes to continuous spin systems with $s \in[-1,1]$. But an analogue of the Uniqueness Theorem 4.5 is lacking here.

6.2. An extension of a method of Lebowitz and Penrose [16] allows us to prove a clustering property of invariant equilibrium states in the region described in Theorem 4.5: if $\bar{A}, \bar{B} \in \bar{B}$ then $\varrho\left(s_{A}+s_{B}\right)-\varrho\left(s_{A}\right) \varrho\left(s_{B}\right)=O\left(e^{-x|a|}\right)$ for suitable $x>0, x$ increasing with the inverse temperature.

In fact one obtains much more precise information about the clustering properties (results for spin $\frac{1}{2}$ case were obtained and reported by the present author in Spring 1973 at École Polytechnique in Paris).

6.3. If one applies a version of the present method to investigate systems at high temperatures one gets uniqueness (part (b) of Theorem 4.5) for all m.f.'s $A$ and the interaction need not be ferromagnetic: for high temperatures the variables are $z_{B}=$ th $K(B)$ which are small for small $|K(B)|$. If one changes the interaction as in Proposition 1.3 then, for any m.f. $A$ the additional terms contain a factor th $\lambda$ which is mall for small $\lambda$. For low temperatures for $\bar{A} \notin \overline{\mathscr{B}}$ one would get additional terms of the form $\exp -2 \lambda$ which are close to 1 for small $\lambda$.

13 It follows from the text that all the ratios of integers in this section have integral values. This refers in particular to $\left(2^{p}-1\right) /(p+1)$ and $\left[2^{p^{2}}+2^{p}-2\right] /(p+1), p+1$-prime. The fact that, for prime $p+1,2^{p^{2}}+2^{p}-2 \equiv 0\left(\bmod p^{2}\right)$ is an amusing corollary of our analysis. 
6.4. The version of the present approach sketched in [13, Section 4.10 (b) gives uniqueness of the invariant equilibrium state in the situations covered by Suzuki and Fisher [2], Theorem 1.

6.5. Estimations of Section 3.6 yield some analyticity and uniqueness for a class of interactions of infinite range, but it is not clear if the domain of analyticity includes in all these cases the low temperature region.

6.6. This paper suggests several problems.

(a) Is it true that for all temperatures

$\Delta^{I} C \Delta^{+}$

or, equivalently, do all invariant equilibrium states, at fixed temperatures, agree on $\left\{s_{A}: \bar{A} \in \overline{\mathscr{B}}\right\}$ ? In this form the question makes sense for non-ferromagnetic systems too.

Let $\tilde{\mathfrak{U}}(K)$ be the subalgebra of $\mathfrak{U}=\mathscr{C}(\mathscr{X})$ generated by $\left\{s_{B}: B \in \mathscr{B}(K)\right\}$. An alternative form of the above problem is: do all invariant equilibrium states at fixed temperature agree on $\tilde{\mathfrak{U}}(K)$ ?

For spin $\frac{1}{2} \tilde{\mathfrak{U}}(K)$ is exactly the linear span of $\left\{s_{A}: \bar{A} \in \overline{\mathscr{B}}\right\}$. But it is not hard to find examples for higher spin where the linear span of $\left\{s_{A}: \bar{A} \in \overline{\mathscr{B}}\right\}$ is strictly larger than $\tilde{\mathfrak{U}}(K)$.

(b) $\Delta^{I}$ is determined by $\overline{\mathscr{B}^{+}}$. On the other hand some information on $\mathscr{B}^{+}$ can be obtained by pure algebra. For instance, in the case of the regular systems of Section 5.6 one can show that $\overline{\mathscr{B}^{+}}$can change at a finite number of points only; this holds presumably in general. That means that, when the temperature is varied, one can parametrize the equilibrium states, in a sense, in a piecewise continuous fashion. It would be helpful to have a good hypothesis on how $\overline{\mathscr{B}^{+}}$ changes with temperature, which elements of $\mathscr{P}_{f}\left(\mathbb{Z}^{\nu}\right)$ appear in $\overline{\mathscr{B}}{ }^{+}$first when the temperature is lowered; for high temperatures $\overline{\mathscr{B}}^{+}=\overline{\mathscr{B}}$ as follows from the uniquess of the equilibrium state.

(c) Problems connected with the results of [6] will be formulated in a paper containing proofs of these results. We mention here only one: is it true that models with external field, or, more generally, such models that $\overline{\mathscr{B}}$ is a principal ideal, have unique invariant equilibrium state at all temperatures?

(d) It can be shown that all spin $\frac{1}{2}$ models on $\mathbb{Z}^{v}$ with two fundamental bonds are self-dual. On the other hand, it is easy to find examples of such models with a large number of phases at low temperatures (cf. Section 5.8), and even with a large number of ideals intermediate between $\overline{\mathscr{B}}^{+}$and $\overline{\mathscr{B}}$. Do all these phases appear at the same temperature given by Kramers-Wannier argument?

\section{References}

1. Dobrushin, R.L.: Gibbsian random fields for lattice systems with pairwise interactions. Funct. Anal. Appl. 2, 44-57 (1968)

2. Suzuki, M., Fisher, M.E.: Zeros of the partition function for the Heisenberg and general Ising models. J. Math. Phys. 12, 235-246 (1971)

3. Ginibre, J.: General formulation of Griffiths' inequalities. Commun. math. Phys. 16, 310-328 (1970) 
4. Griffiths, R. B.: Rigorous results for Ising ferromagnets of arbitrary spin. J. Math. Phys. 10, 1559-1565 (1969)

5. Holsztyński,W.: Precise generators of $A[G]$-modules. Preprint, Institute for Advanced Study

6. Holsztyński, W., Slawny, J.: Phases of ferromagnetic spin systems at low temperatures. Lett. Nuovo Cimento 13, 534-538 (1975)

7. Israel, R.: Thesis, Princeton University

8. Lanford,O.E., Ruelle,D.: Observables at infinity and states with short range correlations in statistical mechanics. Commun. math. Phys. 13, 194-215 (1969)

9. Leff, M.: Proof of third law of thermodynamics for Ising ferromagnets. Phys. Rev. A 2, 2368-2370 (1970)

10. Merlini,D., Gruber,C.: Spin $\frac{1}{2}$ lattice systems: group structure and duality relations. J. Math. Phys. 13, 1814-1823 (1972)

11. Ruelle, D.: Statistical mechanics rigorous results. New York: Benjamin 1969

12. Extension of the Lee-Yang circle theorem. Phys. Rev. Letters 26, 303-304 (1971)

13. Slawny, J.: Analyticity and uniqueness for spin $\frac{1}{2}$ classical ferromagnetic lattice systems at low temperatures. Commun. math. Phys. 34, 271-296 (1973)

14. Slawny, J.: A family of equilibrium states relevant to low temperature behaviour of $\operatorname{spin} \frac{1}{2}$ classical ferromagnets. Breaking of translation symmetry. Commun. math. Phys. 35, 297--305 (1974)

15. Wegner, F. J.: Duality in generalized Ising models and phase transitions without local parameters. J. Math. Phys. 12, 2259-2272 (1971)

16. Lebowitz, J.L., Penrose, O.: Analytic and clustering properties of thermodynamic functions and distribution functions for classical lattice and continuum systems. Commun. math. Phys. 11, 99-124 (1968)

17. Ruelle,D.: Some remarks on the location of zeros of the partition function for lattice systems. Commun. math. Phys. 31, 265-277 (1973)

18. Hörmander, L.: An introduction to complex analysis in several variables, Corollary 1.2.6. Amsterdam: North Holland 1973

19. Lanford,O.E., Robinson,D.: Statistical mechanics of quantum spin systems. III. Commun. math. Phys. 9, 327-338 (1968)

20. Gruber, C., Hintermann, A., Merlini, D.: Analyticity and uniqueness of the invariant equilibrium states for general spin $\frac{1}{2}$ classical lattice systems. Commun. math. Phys. 40, 83-95 (1975)

21. Runnels, L.K.: Zeros of the grand partition function. In: Quantum statistical mechanics in the natural sciences. B. Kursunoglu, S. L. Mintz, S. M. Widmayer (eds.). New York: Plenum Publishing Corporation

22. Freasier, B. C.: High-temperature analyticity of the Widom-Rowlison model with finite repulsion. Phys. Rev. B 8, 2126-2127 (1973)

23. McKean,H.P.: Kramers-Wannier duality for the 2-dimensional Ising model as an instance of Poisson's summation formula. J. Math. Phys. 5, 775-776 (1964)

24. Holsztyński, W., Slawny, J.: Phase transitions in ferromagnetic spin systems at low temperatures. Preprint

Communicated by G. Gallavotti

Received April 12, 1975

Note Added in Proof. A proof of a strengthened version of Theorem 5.6 is contained in [24]; the regularity assumption is not needed if g.c.d. $\mathscr{B}=1$ (as in examples of Sect. 5.8.). 
